\title{
Study of Paracetamol Infusion as Pre-Emptive Analgesic in Lower Abdominal Surgeries
}

\author{
Divya Vincent ${ }^{1}$ Vishma $\mathrm{K}^{2}$ \\ Department of Anesthesiology, Father Muller Medical College/ Rajiv Gandhi University, India ${ }^{I}$ \\ Department of Anesthesiology, Father Muller Medical College/ Rajiv Gandhi University, India ${ }^{2}$
}

\begin{abstract}
:
Introduction: Pre-emptive analgesia is widely used to control intraoperative and postoperative pain. Intravenous Paracetamol, a non opioid analgesic gained wide popularity as to control pain during surgery. Present study is undertaken to check whether intravenous Paracetamol can be used as pre-emptive analgesic in patients undergoing lower abdominal surgeries under general anaesthesia.

Methodology: 60 patients of ASA grade I \& II undergoing lower abdominal surgeries under general anaesthesia were included in the study. Patients in the study group $(n=30)$ received Paracetamol infusion (1000mg./100ml.) 15 minutes prior to induction of anaesthesia. All patients received uniform type of general anaesthesia and intra-operative fentanyl $2 \mathrm{mcg} . / \mathrm{kg}$. Monitoring includes Heart Rate (HR), Oxygen Saturation(SPO2), Blood Pressure. In the post operative period, pain is assessed at an interval of every 30 minutes till the first rescue analgesic. Pain is assessed using Visual Analogue Scale (VAS). The end point of the study is when rescue analgesic is administered to the patient.

Result: Post operatively mean VAS score was better in the Paracetamol group compared to control group. Time requirement of first dose of rescue analgesic in the postoperative period was significantly prolonged in the Paracetamol group.

Conclusion: It was concluded that IV Paracetamol is beneficial as a pre-emptive analgesic in patients who undergo lower abdominal surgeries.
\end{abstract}

Keywords: Paracetamol, pre-emptive analgesia, general anesthesia

\section{Introduction}

Pain can be classified as nociceptive, inflammatory or neuropathic pain. Postoperative pain is nociceptive.Nociceptive pain is a transient pain occuring due to noxious stimuli.Postoperative pain is considered a form of acute pain due to the surgical trauma with an inflammatory reaction, an initiation of an afferent neuronal barrage ${ }^{1}$. Proper pain relief is necessary for a better outcome. Effective postoperative pain relief is now considered pivotal in successful care of post surgical patients.

Pre-emptive analgesia is the treatment that is initiated before the surgical procedure in order to reduce the sensitization of peripheral and central pain pathways. The concept of pre-emptive analgesia was formulated by Crile $^{2}$. Owing to this protective effect on the nociceptive system, pre-emptive analgesia has the potential to be more effective than a similar analgesic treatment initiated after surgery ${ }^{3}$. Surgery offers the most promising setting for pre-emptive analgesia because the timing of noxious stimuli is known.

Paracetamol is a safe and cost effective non opioid centrally acting analgesic. Paracetamol has the advantage of being the only non opioid analgesic available in oral,rectal or intravenous formulations. The intravenous formulation is advantageous in post surgical situations when oral(eg. Infection with severe fever or vomiting) or rectal(eg.high variability in uptake or bioavailability)routes are not suitable or effective. The aim of this study is to see the effects of Paracetamol infusion as a pre-emptive analgesic in lower abdominal surgeries as well as the influence of this drug on intraoperative requirement of narcotic analgesia

Our aim was to check whether Paracetamol provides analgesia in the post operative period when administered pre-operatively,study the duration of analgesia, timing of first rescue analgesic administration \& the amount of rescue analgesics needed.

\section{Headings}

\section{Introduction}

\section{Materials and methods}

1.1 Sampling procedure and size

1.2 Inclusion criteria

1.3 Exclusion criteria

1.4 Methodology 


\section{Statistical analysis \\ 4. Results \\ 5. Discussion}

\section{Sampling procedure and size}

\section{Materials And Methods}

Patients in Yenepoyaand Father mullerMedical College Hospital, Mangalore who are admitted during the period of 1/10/14 to 1/9/15 undergoing lower abdominal surgeries under general anaesthesia.

Size of study group :60

Method of collection of data

\section{Inclusion criteria:}

- Patients undergoing surgery under GA.

-ASA grade 1 and 2.

-Patients of either gender between age 18-65 year.

-Weighing 40-70kg.

-Surgeries lasting less than 3-4 hours

\section{Exclusion criteria:}

- Paediatric age group.

- $\quad$ ASA grade 3 and 4.

- Procedure done under regional anesthesia.

- Patients having liver disease.

- $\quad$ Past H/O allergy to Paracetamol.

\section{Method:}

After informed consent from patients, they received the following premedication on the night prior to surgery -Tab Lorazepam 1mg,Tab Ranitidine 150mg On the morning of surgery, patients were shifted to preoperative holding room, Monitors $\mathrm{SpO}_{2}$, ECG, NIBP were connected, $1 \mathrm{~g}$ IV Paracetamol infusion started 15 mins prior to surgery in the study group $(\mathrm{n}=30)$. All patients received general anaesthesia as follows Preoxygenation with $100 \%$ oxygen for 3 mins, Inducing agent: Propofol, Intraop Opioid - Fentanyl $1 \mathrm{mcg} / \mathrm{kg}$, Long acting muscle relaxant: Vecuronium intubation\& maintenance with $66 \% \mathrm{~N}_{2} \mathrm{O}$ and $33 \% \mathrm{O}_{2}$ Intraoperatively the following parameters were monitored SpO2 ,Heart rate BP (NIBP), systolic, diastolic and mean BP. In case of additional requirement of analgesic intraoperatively, it was noted. In the post operative period, pain was assessed in the patient at an internal of every 30 mins till the first rescue analgesic. Pain was assessed using Visual Analogue Scale (VAS) ${ }^{5}$ A major advantage of the VAS as a measure of sensory pain intensity is its ratio scale properties ${ }^{6}$, minimal intrusiveness and conceptual simplicity. End point of study was when patient requests for medication for pain.

\section{Statistical analysis}

Power analysis from similar studies suggest that a sample size of 30 patients/group is required to get the power of study to $80 \%$, with 0.05 level of significance. All the data was fed into the IBM SPSS software , mean and standard deviation was used for continuous data and median for non parametric data. The two groups were compared using analysis of variance (ANOVA). The sedation was compared with the help of Wilcoxon rank-sum test/ $t$ test. $\mathrm{P}$ values of $<0.05$ and Confidence intervals of $>95$ if achieved will be considered significant.

\section{Results}

The mean weight of the two groups was compared- Table 1

With a $\mathrm{p}$ value of 0.944 , the weights of patients within both the groups do not any statistical significance and are comparable.

The mean duration of surgery in both groups was compared- Fig. 2

Comparing the duration of surgery, the IV Paracetamol group had a mean duration of 144.00 and the control group had a mean of 135.466 . With a $p$ value of 0.265 , there was no significant statistical difference between the two groups

The total number of males and females included in i.vParacetamol group and control group were the same. Hence both groups were comparable 
Patients in both IV Paracetamol group and control group required additional Fentanyl intraoperatively .Even though less number of patients required intraoperative Fentanyl, this did not achieve any statistical significance $(\mathrm{p}=0.08)$-Table 2

In both groups the VAS scores at specific post operative time intervals of 30 mins, 60 mins, 90 mins, 120 mins,240mins and 360 mins were compared. The mean of VAS score at 30 mins and 60 mins was 2.033 and 2.466 respectively in the ivParacetamol group as compared to 4.366 and 4.133 in the control group respectively. This was statistically significant with a $\mathrm{p}$ value of $<0.001$. The mean time for rescue analgesic was 240 mins post-operatively as compared to $64 \mathrm{~min}$ in the control group which was statistically very significant- Fig 4

\section{Side effects:}

7 patients in the ivParacetamol group and 11 among the control group had side effects like dizziness, nausea, vomiting, but did not attain any statistical significance.

\section{Discussion}

Pain is a consistent and predominant complaint among individuals following most surgical interventions ${ }^{7}$. Surgery produces tissue injury with consequent release of histamine and inflammatory mediators such as peptides(e.g., bradykinin ),lipids(e.g., prostaglandins), neurotransmitters (e.g.,serotonin) and neurotropins (e.g., nerve growth factor) ${ }^{8}$ The definition of pre-emptive analgesia has been recently reviewed by Kissin ${ }^{9}$. Pre emptive analgesia has been defined as :(1) antinociceptive treatment starting before surgery or (2) antinociceptive treatment that prevents central sensitisation. In our study, both the groups were comparable with respect to age, sex distribution, weight and duration of surgery.

\section{Introperative requirement of fentanyl (additional):}

Patients in both IV Paracetamol group and control group required additional Fentanyl intraoperatively, based on the weight of the patient a duration of surgery.

\section{Time of injection of additional Fentanyl intraoperatively:}

In the IV Paracetamol group, the mean intraoperative time at which additional Fentanyl was required was 144 mins, whereas in the control group it was at a mean of 129 mins. With a p value of 0.013 it was found to be statistically significant.It can be concluded that analgesia provided by IVParacetamol was for a longer duration than Fentanyl alone.

\section{Time of requirement of first rescue analgesic:}

The mean time of requirement of rescue analgesic was 240 mins post operatively, as compared to 64 mins in the control group. This was statistically significant with a p value of 0.001 . Side effects in our study:7 patients in the iv Paracetamol group and 11 among the control group had side effects like dizziness, nausea, vomiting, but did not attain any statistical significance.

\section{Post operative mean VAS score in our study:}

In both groups the VAS scores at specific post operative time intervals of $30 \mathrm{mins}, 60 \mathrm{mins}, 90 \mathrm{mins}$, 120 mins,240mins and 360 mins were compared. The mean of VAS score at 30 mins and 60 mins was 2.033 and 2.466 respectively in the i.vParacetamol group as compared to 4.366 and 4.133 in the control group respectively. All other time intervals, the mean of VAS scores in both groups was found to be comparable.

\section{Figures and Tables}

Tab 1: Comparison of weight distribution

\begin{tabular}{|l|l|l|l|l|l|}
\hline & Group & $\mathbf{N}$ & Mean (wt in kg) & Std. Deviation & t \\
\hline & Pct & 30 & 56.9000 & 10.10411 & .07000 \\
\cline { 2 - 6 } & Controls & 30 & 56.7000 & 11.93127 & $\mathrm{p}=.944 \mathrm{~ns}$ \\
\hline
\end{tabular}

Pct- Paracetamol 


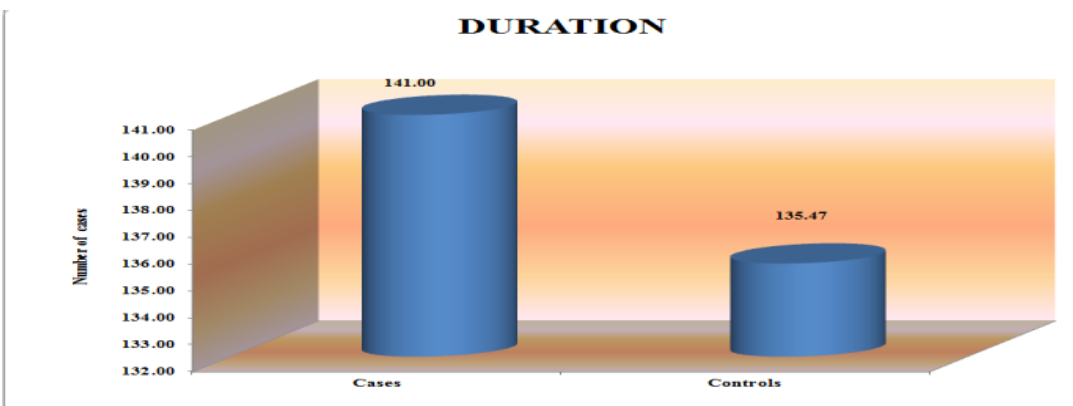

Fig 1: Comparison of duration of surgery

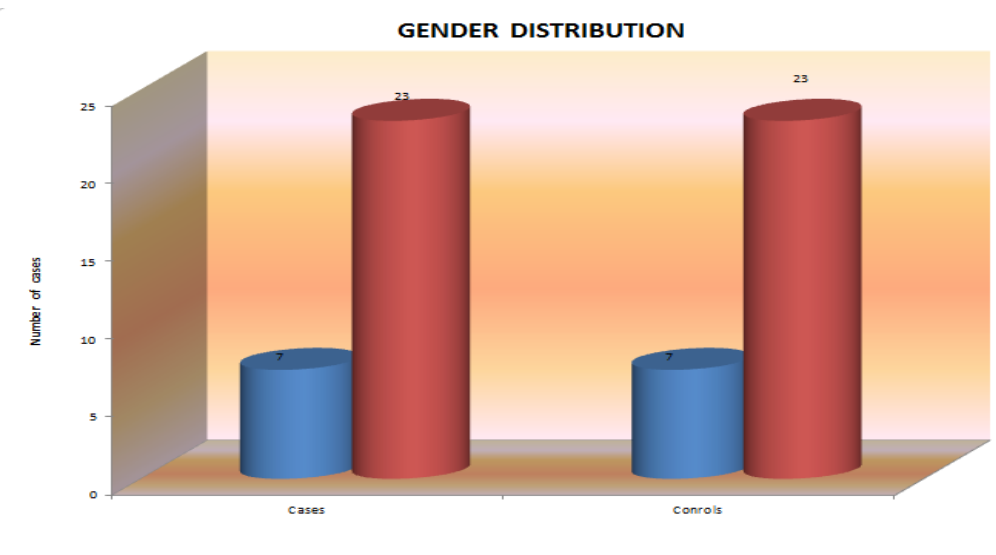

Fig. 2: Comparison of sex distribution

Tab 2: Comparison of intraoperative requirement of additional fentanyl

\begin{tabular}{|l|l|l|l|l|l|}
\hline \multicolumn{3}{|c}{} & Group & Total \\
\cline { 3 - 5 } \multicolumn{2}{|c|}{ Fentanyl } & \multirow{2}{*}{.00} & Pct & Controls & \\
\cline { 3 - 6 } & & $\%$ & $83.3 \%$ & $63.3 \%$ & $43.3 \%$ \\
\cline { 3 - 6 } & \multirow{2}{*}{1.00} & Count & 5 & 11 & 16 \\
\cline { 3 - 6 } & & $\%$ & $16.7 \%$ & $36.7 \%$ & $26.7 \%$ \\
\hline \multirow{2}{*}{ Total } & Count & 30 & 30 & 60 \\
\cline { 3 - 5 } & $\%$ & $100.0 \%$ & $100.0 \%$ & $100.0 \%$ \\
\hline
\end{tabular}

$\mathrm{X}^{2}=3.068 \mathrm{p}=.08 \mathrm{~ns}$

Pct-Paracetamol

Tab 3:Time of injection of additional fentanyl intraoperatively

\begin{tabular}{|l|l|l|l|l|l|}
\hline \multicolumn{7}{|c|}{ Time of njection } \\
\hline \multirow{2}{*}{} & Group & $\mathbf{N}$ & $\begin{array}{l}\text { Mean } \\
\text { (time in mins) }\end{array}$ & $\begin{array}{l}\text { Std. } \\
\text { Deviation }\end{array}$ & $\mathbf{t}$ \\
\hline & Pct & 5 & 144.000 & 8.2158 & 2.8440 \\
\cline { 2 - 7 } & Controls & 11 & 129.545 & 9.8627 & $\mathrm{p}=.013 \mathrm{sig}$ \\
\hline
\end{tabular}

Pct-paracetamol

vaspst- visual analogue scale post surgical time 
Study Of Paracetamol Infusion As Pre-Emptive Analgesic In Lower Abdominal Surgeries.

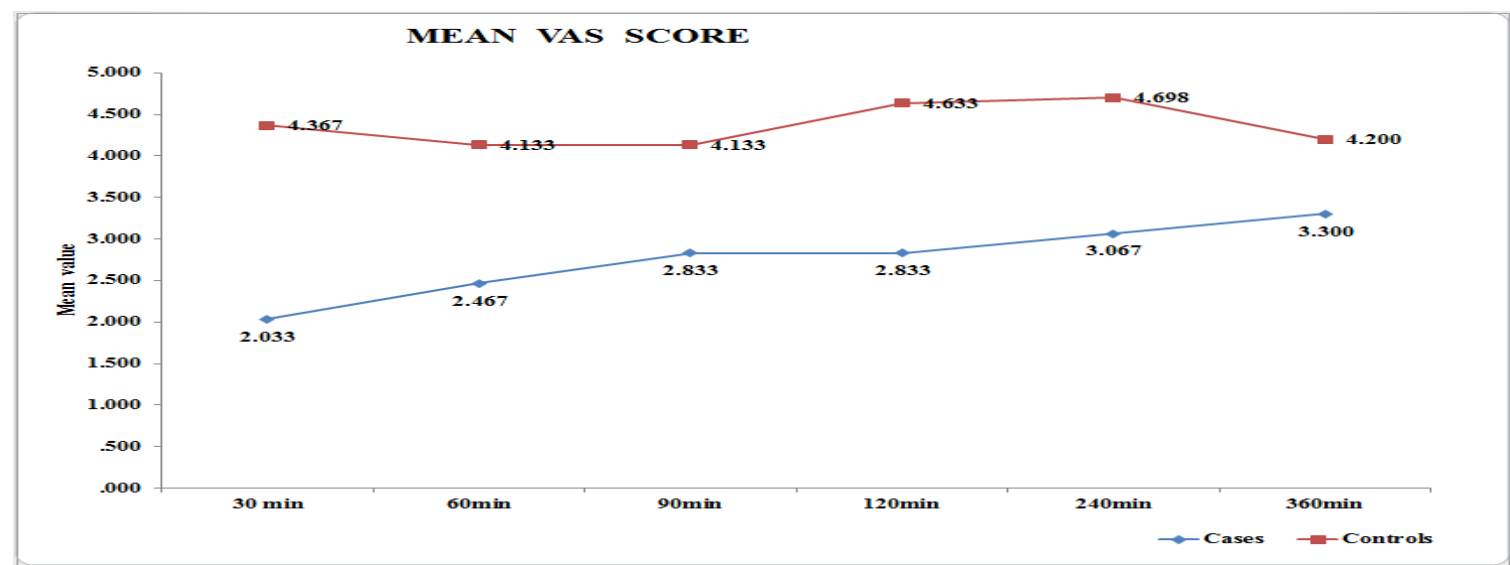

Fig 3: Comparison of Mean VAS score

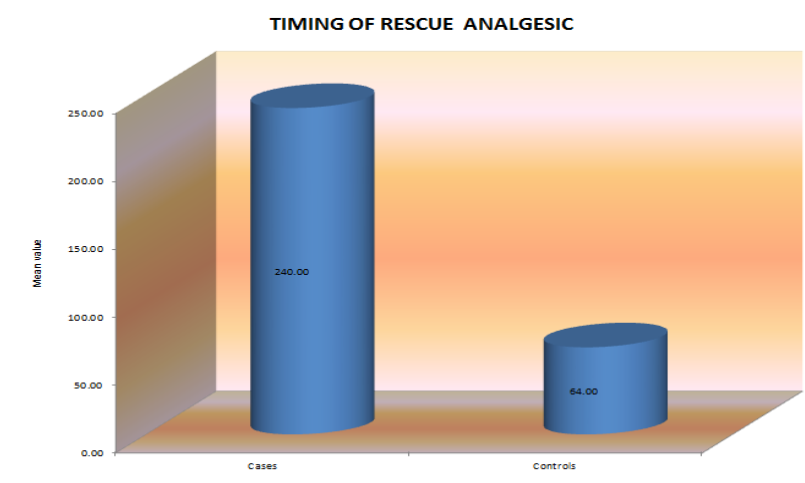

Fig 4:Comparison of time of requirement of first rescue analgesic

Tab 4: Side effects

\begin{tabular}{|c|c|c|c|c|c|}
\hline \multicolumn{2}{|c|}{ Side effect } & \multicolumn{2}{|c|}{ Group } & \multirow{2}{*}{ Total } \\
\cline { 4 - 6 } \multicolumn{2}{|c|}{} & Pct & Controls & \\
\hline \multirow{3}{*}{ Side effect } & \multirow{2}{*}{ No } & Count & 23 & 19 & 42 \\
\cline { 3 - 6 } & & $\%$ & $76.7 \%$ & $63.3 \%$ & $70.0 \%$ \\
\cline { 3 - 6 } & \multirow{2}{*}{ Yes } & Count & 7 & 11 & 18 \\
\cline { 3 - 6 } & & $\%$ & $23.3 \%$ & $36.7 \%$ & $30.0 \%$ \\
\hline \multirow{2}{*}{ Total } & Count & 30 & 30 & 60 \\
\cline { 3 - 5 } & & $\%$ & $100.0 \%$ & $100.0 \%$ & $100.0 \%$ \\
\hline
\end{tabular}

$\mathrm{X}^{2}=1.27 \mathrm{p}=.26 \mathrm{~ns}$ pct-paracetamol

\section{Conclusion}

It can be concluded that IV Paracetamol is beneficial as a pre-emptive analgesic in patients who undergo lower abdominal surgeries. No significant side effects were seen.

\section{References}

[1]. Jorgen and B Dhal and HenrikKehlit "Post operative pain and its management". Text book of pain-Wall and Melzack 5th edition.

[2]. Merriam Webster Dictionary

[3]. "ISAP definition, full entry". Retrieved 6 October 2009. This often quoted definition was first formulated by the ISAP subcommittee on Taxonomy, The need for taxonomy. Pain 6(3): 247-252.

[4]. Choinere M, Mezlac R, Girard N, Rondean J, Paquin M J. "Comparison between patients and nurses assessment of pain and medication efficacy in severe burns injuries" Pain 1990;40;143-152.

[5]. Price DD, McGrath PA, Rafii A, Buckingham B. "The validation of Visual Analogue Scale as ratio scale measure for chronic and experimental pain" Pain 1983.17;45-46.

[6]. David James and Douglas. "Acute post operative pain" Wylie and Churchill-Davidson-a practice of Anaesthesia-7th edition

[7]. Julius D, Bashbaum A L: "Molecular mechanics of Nociception". Nature 2001; 413;203

[8]. Kissin I "Preemptive analgesia". Anaesthesiology 2000; 93: 1138-43

[9]. Sean T. Duggan and Lesley J. Scott Wolters Kluwer Health Adis, Auckland, New Zealand, Intravenous Paracetamol (Acetaminophen) Drugs 2009; 69 (1): 101-113 